\title{
Activation of inflammasome signaling mediates pathology of acute $P$. aeruginosa pneumonia
}

\author{
Taylor S. Cohen ${ }^{1}$ and Alice S. Prince ${ }^{1,2}$
}

${ }^{1}$ Department of Pediatrics and 2Department of Pharmacology, College of Physicians and Surgeons, Columbia University, New York, New York, USA.

\begin{abstract}
The respiratory tract is exceptionally well defended against infection from inhaled bacteria, with multiple proinflammatory signaling cascades recruiting phagocytes to clear airway pathogens. However, organisms that efficiently activate damaging innate immune responses, such as those mediated by the inflammasome and caspase-1, may cause pulmonary damage and interfere with bacterial clearance. The extracellular, opportunistic pathogen Pseudomonas aeruginosa expresses not only pathogen-associated molecular patterns that activate NF-KB signaling in epithelial and immune cells, but also flagella that activate the NLRC4 inflammasome. We demonstrate that induction of inflammasome signaling, ascribed primarily to the alveolar macrophage, impaired $P$. aernginosa clearance and was associated with increased apoptosis/pyroptosis and mortality in a murine model of acute pneumonia. Strategies that limited inflammasome activation, including infection by fliC mutants, depletion of macrophages, deletion of NLRC4, reduction of IL- $1 \beta$ and IL-18 production, inhibition of caspase-1, and inhibition of downstream signaling in IL-1R- or IL-18R-null mice, all resulted in enhanced bacterial clearance and diminished pathology. These results demonstrate that the inflammasome provides a potential target to limit the pathological consequences of acute $P$. aeruginosa pulmonary infection.
\end{abstract}

\section{Introduction}

The innate immune response to inhaled bacteria is a complex, highly regulated set of signaling events that ideally result in pathogen clearance without damage to the lung. Airway epithelial cells, resident immune cells (such as alveolar macrophages [AMs] and DCs), and recruited neutrophils, macrophages, DCs, and $T$ cells all participate in the initial response to bacterial contamination of the lower airways. Given the ubiquity of exposure to potential pathogens in the environment and the frequency of aspiration of upper airway flora, these immune defenses are usually quite efficient. However, after significant aspiration of bacteria such as Pseudomonas aeruginosa, as occurs in a hospitalassociated setting, severe pneumonia associated with significant mortality often results $(1,2)$.

There is substantial redundancy in the signaling activated in response to pathogen-associated molecular patterns (PAMPs) released by Gram-negative rods in the airway lumen. Host cells respond to these ligands via the apical display of TLRs and induction of NF-кB-mediated proinflammatory gene expression that includes IL-6, IL-8, and GM-CSF (3-7). Shed components of $P$. aeruginosa, including flagella (TLR5), LPS (TLR4), and cell wall lipoproteins (TLR2), are immunostimulatory to varying extents, even if few intact bacteria gain direct access to the epithelial surface (8-13). After priming by LPS or other PAMPs, activation of the NLR family CARD domain-containing protein 4 (NLRC4) inflammasome by internalized flagellin stimulates caspase-1, resulting in pyroptosis - a highly proinflammatory mode of cell death associated with tissue damage - and release of inflammatory cytokines, such as IL-1 $\beta$ and IL-18 (14-22). Both IL-1 $\beta$ and IL-18 have been identified as mediators of local inflammation in the lung and tissue remodeling systemically $(1,2,23-29)$.

Conflict of interest: The authors have declared that no conflict of interest exists. Citation for this article: J Clin Invest. 2013;123(4):1630-1637. doi:10.1172/JCI66142.
The responses of various transgenic and knockout mice demonstrate that rapid recruitment of neutrophils into the lung is essential in clearing $P$. aeruginosa infection (3-7, 30-33). However, these studies do not address whether specific signaling events are associated with pathological responses in the lung. We postulated that activation of inflammasome signaling might be deleterious in the eventual outcome of acute $P$. aeruginosa pneumonia. The experiments described herein demonstrated that caspase- 1 cleavage of pro-IL-1 $\beta$ and pro-IL-18 - primarily in AMs after NLRC4 inflammasome activation by flagellin - was associated with excessive pathology and that production of these cytokines was regulated by type I IFN signaling. In the setting of acute $P$. aeruginosa pneumonia, inhibiting inflammasome signaling significantly improved outcome.

\section{Results}

Flagellin is the major stimulus for $I L-1 \beta$ production in acute $P$. aeruginosa pneumonia. Extracellular bacterial pathogens express numerous PAMPs that evoke proinflammatory signaling. Flagella, in addition to activating NF- $\kappa \mathrm{B}$ via TLR5, function as ligands for nonopsonic phagocytosis, providing an efficient mechanism for internalization of bacteria and delivery of flagellin to the inflammasome $(8-13,34)$. We compared the consequences of infection with the $P$. aeruginosa strain PAK (an isolate whose WT form is motile and expresses several type III-secreted toxins), fliC PAK mutants (which do not express flagella), and motAB PAK mutants (which express flagella, but do not swim) (14-22, 35). Although fliC PAK was not as effectively cleared from the airway as motAB PAK and WT PAK $(P<0.05$ and $P<0.01$, respectively), equivalent numbers of each strain were found in the lung tissue (Figure 1, A and B). The fliC mutants were associated with reduced mortality compared with mot $A B$ and WT PAK (Figure 1C) and resulted in less pathology and less positive staining for TUNEL (Figure 1D), a marker of both apoptotic and pyroptotic cell death (36). Furthermore, overall 
A
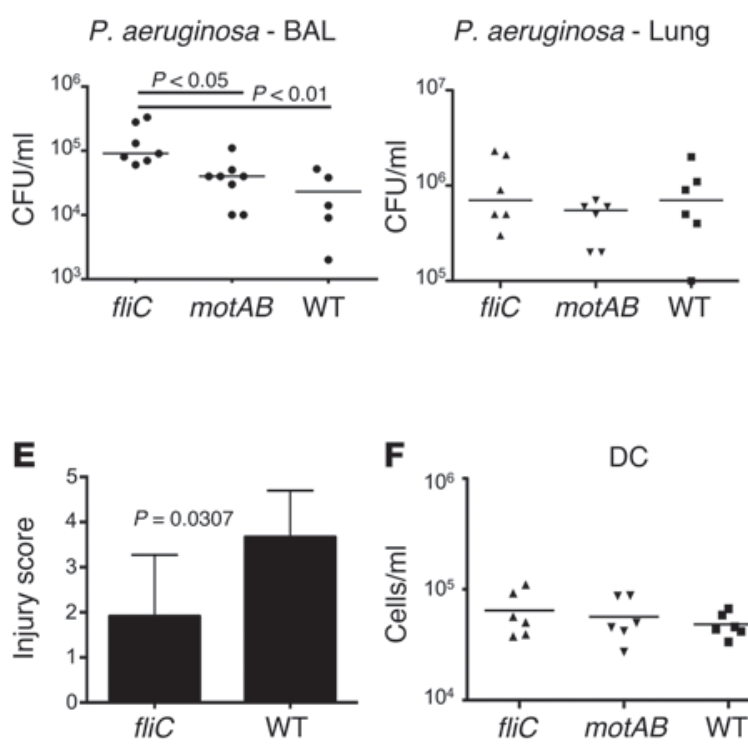

G

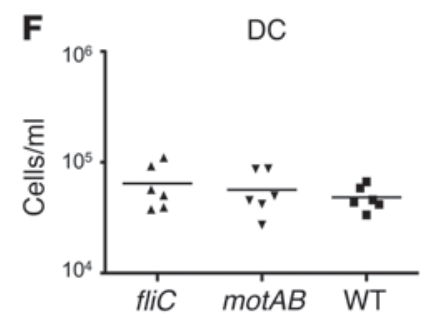

H
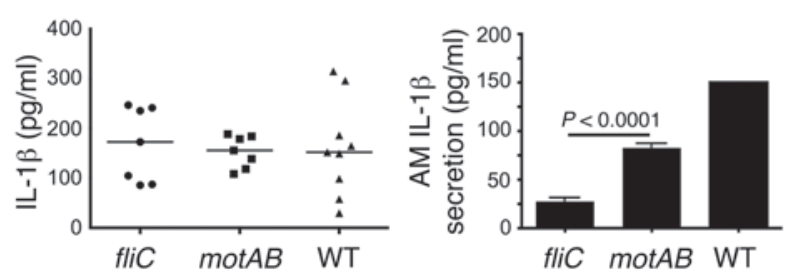

C

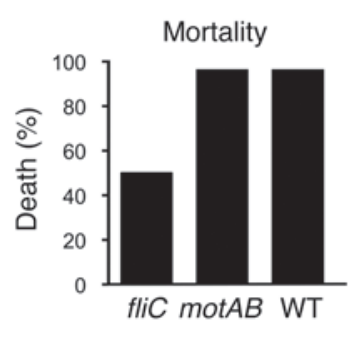

D
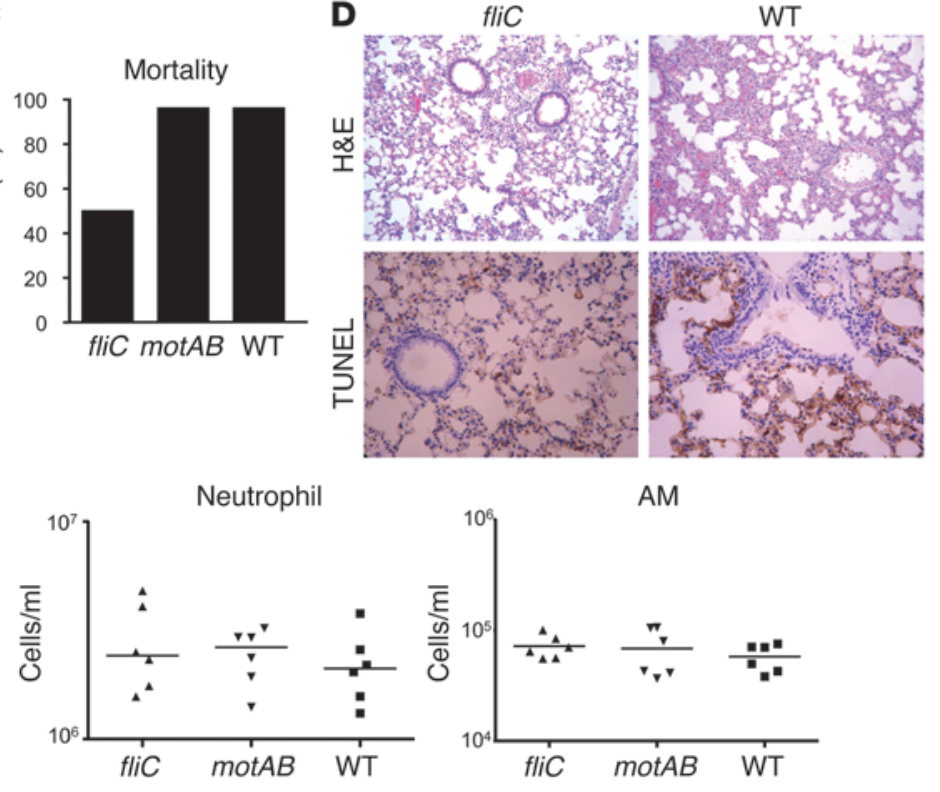

I

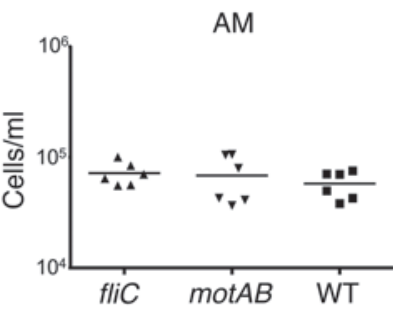

J Cleavage of pro-Caspase-1 WT fliC motAB
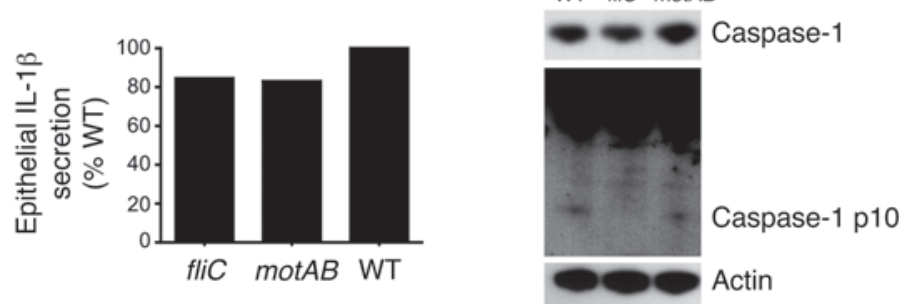

Figure 1

Role of flagella in P. aeruginosa infection. (A and B) Numbers of fliC, motAB, and WT PAK recovered from (A) BAL and (B) lung tissue. (C) Mortality 18 hours after infection $\left(10^{8} \mathrm{CFU} /\right.$ mouse; $n=8$ ). (D) H\&E (original magnification, $\left.\times 100\right)$ and TUNEL (original magnification, $\left.\times 400\right)$ staining of PAK-infected lungs. (E) Lung injury score. (F) FACS analysis of immune cell populations - neutrophils, AMs, and DCs - in BAL. (G) IL-1 $\beta$ levels in BAL. (H) IL-1 $\beta$ secreted from AMs in vitro. (I) IL-1 $\beta$ secreted from primary murine nasal epithelial cells in vitro. Levels are normalized to WT. (J) Cleavage of pro-caspase-1 in lung tissue of WT, fliC, and motAB PAK-infected mice. Data in A-I are from at least 2 independent experiments.

lung injury was significantly reduced in lungs infected with fliC compared with WT PAK $(P=0.0307$; Figure 1E). Equivalent numbers of immune cells were recruited to the lung in response to infection with fliC, motAB, and WT PAK (Figure 1F). No difference in the level of IL-1 $\beta$ was observed in the airways of mice infected with $f l i C, \operatorname{mot} A B$, and WT PAK; however, flagellin-induced production of IL-1 $\beta$ was associated with AMs, not epithelial cells, which produced similar amounts of IL- $1 \beta$ in response to fliC and mot $A B$ PAK (Figure 1, G-I). Processing of pro-caspase-1 in response to PAK was dependent on the presence of flagellin in homogenized lung tissue (Figure 1J). Thus, P. aeruginosa expression of flagellin, while necessary for optimal bacterial clearance from the airway, was nonetheless associated with caspase- 1 activation, macrophage production of IL-1 $\beta$, increased tissue damage, and mortality.

Depletion of AMs decreases IL-1 $\beta$ signaling and improves $P$. aeruginosa clearance. The results in Figure 1 suggest that activation of the inflammasome in response to PAK flagella correlates with increased mortality and pathology or impaired bacterial clearance. Since IL- $1 \beta$ secretion from the AM, not epithelial cells, was dependent on the presence of flagella, we hypothesized that depletion of AMs would improve outcome from acute PAK infection. We used clodronate- loaded liposomes to deplete AMs in the bronchoalveolar lavage (BAL); PBS-loaded liposomes served as a control. Clodronate was observed to deplete $83.5 \%$ of the AM population in uninfected mice (Figure 2A). In infected animals, AM depletion by clodronate was observed in the airway and lung tissue $(P=0.0062$ and $P=0.0379$, respectively; Figure 2, B and C). Numbers of DCs and neutrophils were not statistically different in either airway or tissue. Depletion of AMs resulted in significantly less IL- $1 \beta$ and IL-18 in the BAL $(P=0.0012$ and $P=0.0140$, respectively), which correlated with significantly improved PAK clearance from the airway $(P<0.0001)$ and a trend toward reduced PAK in lung tissue (Figure 2, D-G). Mortality was significantly reduced in AM-depleted animals $(P=0.001)$, which did not correlate with increased dissemination of PAK to the spleen (Figure 2, H and I). AM-depleted mice showed less lung pathology than did controls, as evidenced by better preserved architecture and air spaces, reduced TUNEL staining, and significantly reduced lung injury score $(P<0.0001$; Figure 2, J and $\mathrm{K})$.

GM-CSF enhances neutrophil activity. We sought to explain how the same number of neutrophils in the airway of PBS and clodronate-treated mice could result in different efficiencies of bacterial clearance. Since increased numbers of PAK were not found in the 
A

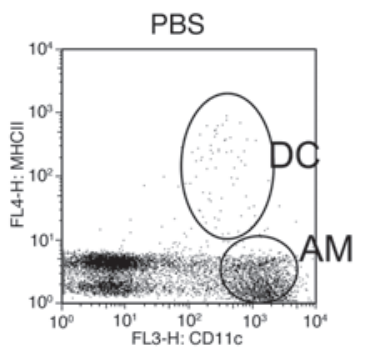

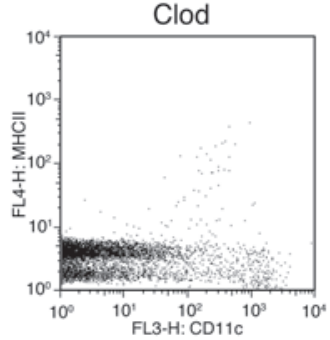

DC - BAL
D

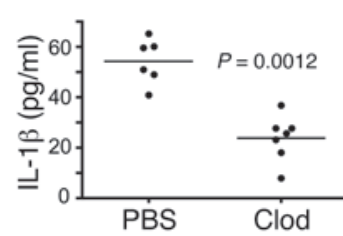

F ${ }_{10^{6}} P$. aeruginosa - BAL
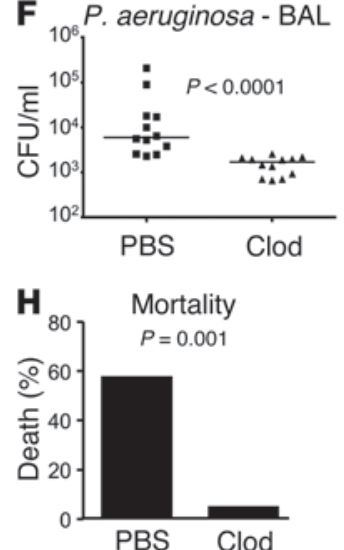

E

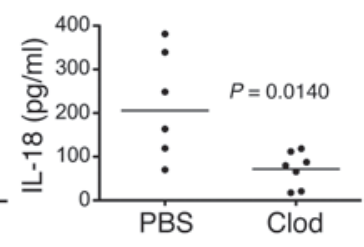

B

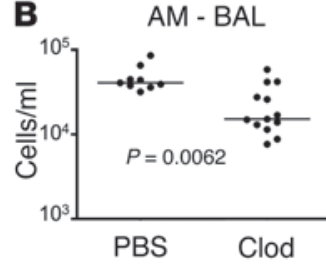

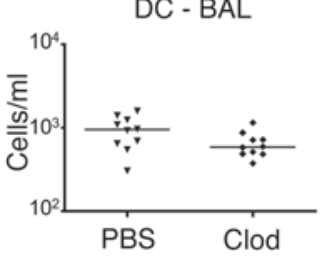

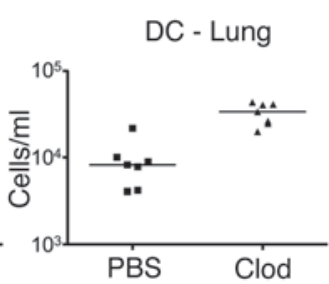

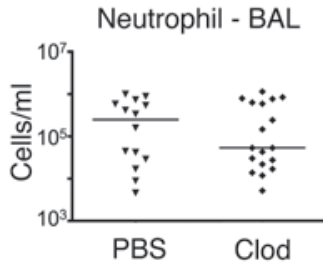

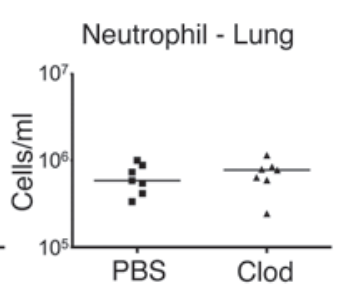

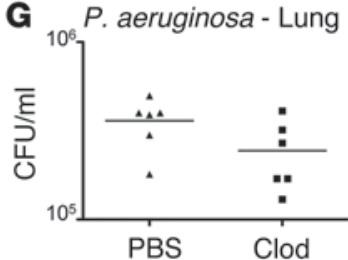

I P. aeruginosa-Spleen

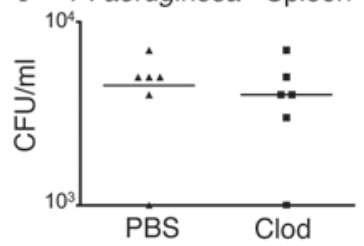

K

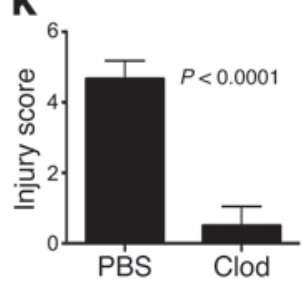

J

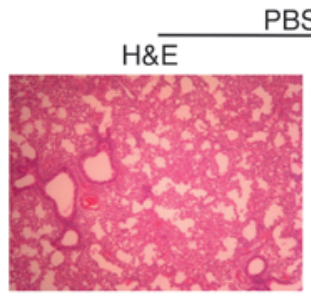

BS-PAK

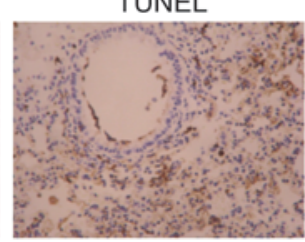

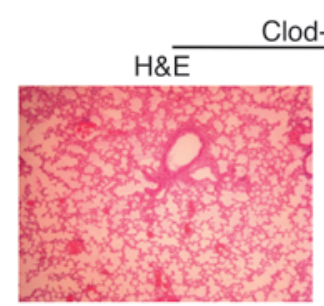

lod-PAK

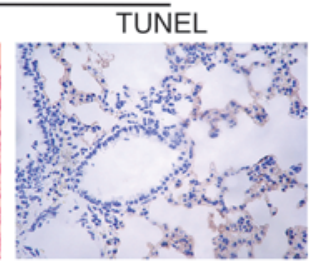

\section{Figure 2}

AMs mediate pathology via IL-1 $\beta$. (A) FACS plots of AMs and DCs from BAL of uninfected mice treated with PBS or clodronate liposome (Clod). (B and C) Immune cell populations in (B) BAL and (C) lungs after infection. (D and E) Levels of (D) IL-1 $\beta$ and (E) IL-18 in BAL. (F and G) Numbers of PAK recovered from (F) BAL and (G) lung. (H) Mortality 18 hours after infection (108 CFU/mouse; $n=12$ per group). (I) Numbers of PAK recovered from spleen. $(\mathbf{J}) \mathrm{H} \& E$ (original magnification, $\times 100)$ and TUNEL (original magnification, $\times 400)$ staining of PAK-infected lungs. $(\mathbf{K})$ Lung injury score. Data are representative of at least 2 independent experiments.

lung tissue or in the spleen in AM-depleted mice, we hypothesized that the phagocytic capacity of neutrophils in the airway of clodronate-treated mice was increased. Indeed, neutrophils in the clodronate-treated group were significantly more phagocytic than controls, as determined by quantifying the amount of ingested GFP-labeled PAK ( $P=0.0268$; Supplemental Figure 1A; supplemental material available online with this article; doi:10.1172/ JCI66142DS1). Airway levels of GM-CSF were significantly increased in clodronate-treated mice $(P=0.0015$; Supplemental Figure $1 \mathrm{~B})$. The neutralization of GM-CSF in clodronate-treated animals reduced phagocytosis of bacteria by neutrophils, but did not increase the numbers of bacteria recovered from BAL (Supplemental Figure 1, C-E). These data suggest that while neutrophil function was increased by compensatory GM-CSF production in clodronate-treated animals, this is not the mechanism responsible for improved bacterial clearance and reduced pathology.

Diminished IL-17 does not impair P. aeruginosa clearance in clodronatetreated mice. In addition to changes in GM-CSF, IL-1 $\beta$, and IL-18, additional chemokines and cytokines, such as chemokine (C-X-C) motif ligand 1 (KC), IL-6, IL-10, and IL-17, were also significantly decreased in clodronate-treated mice $(P<0.05$ for all comparisons; Supplemental Figure 2). Importantly, AM depletion did not alter levels of IL-1Ra, an endogenous inhibitor of IL-1 $\beta$. The participation of IL- 6 and IL-17 in the host response to extracellular pathogens has been well established (37-41). There were similar numbers of CD11 ${ }^{-}$ $\mathrm{CD}^{+}$cells in BAL of clodronate-treated animals; however, fewer of these were $\mathrm{IL}-17^{+}$, correlating with substantially decreased phosphoSTAT3 in BAL cells (Supplemental Figure 3, A-C). To determine whether the decreased IL-17 signaling affected bacterial clearance, we treated mice with either anti-IL-6 or anti-IL-17 and monitored the level of infection in the lung compared with mice treated with an IgG control (Supplemental Figure 3, D and E). Whereas anti-IL-6 did not alter PAK clearance, neutralization of IL-17 resulted in increased retention of PAK in the mouse airway. Similar results were obtained from $C d 4^{-/-}$mice $(P=0.0159$; Supplemental Figure 3F). Thus, IL-17 has beneficial effects on bacterial clearance, and the clodronate-treated mice, despite diminished IL-17, were still better able to clear PAK from the airways than the untreated mice. 

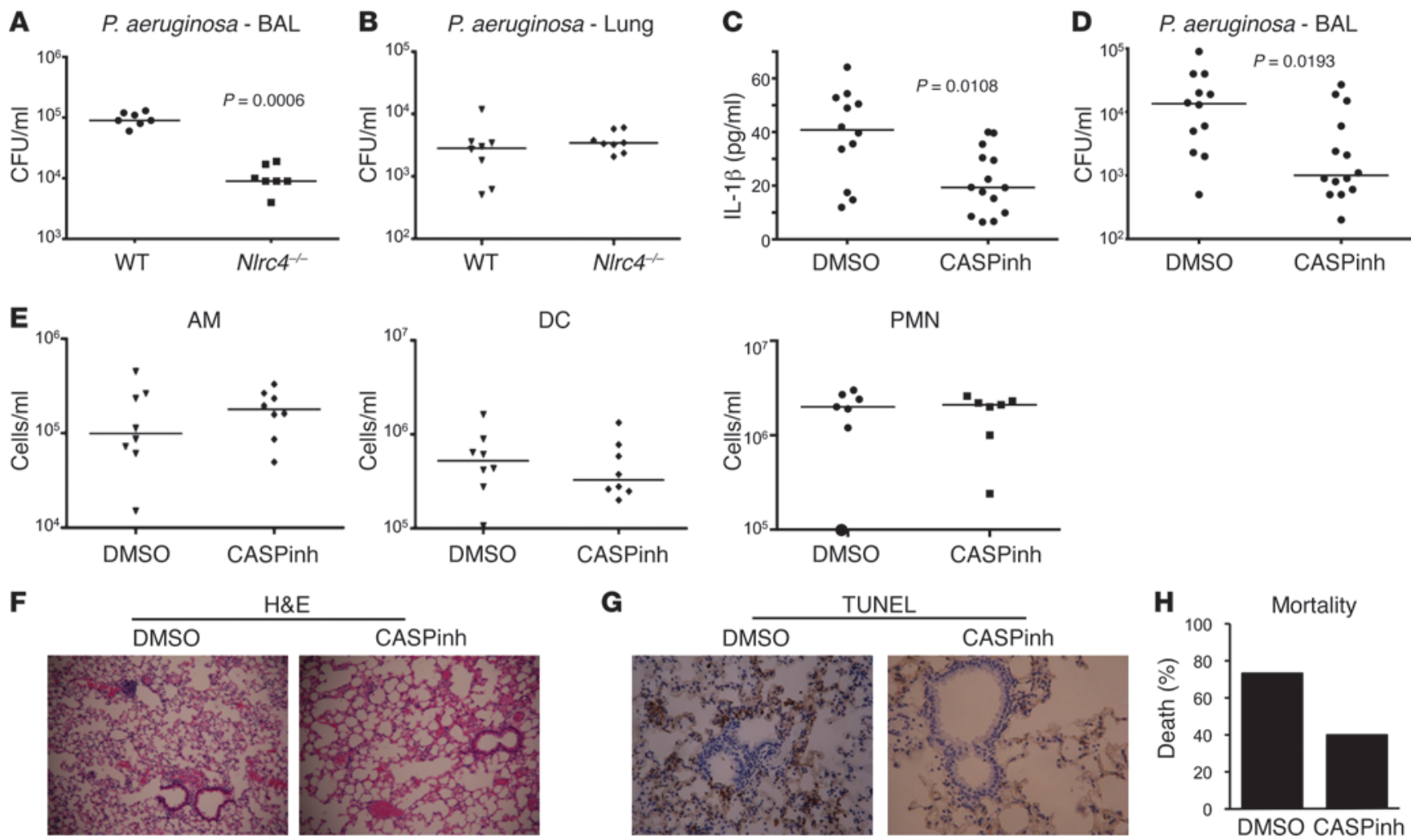

H\&E
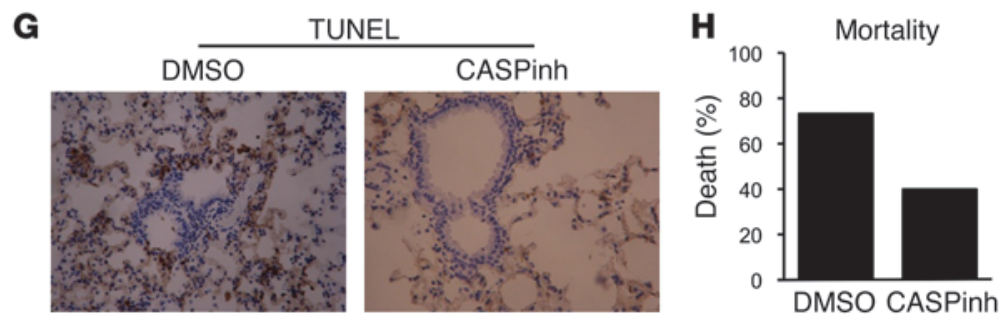

Figure 3

Role of the NLRC4 inflammasome in P. aeruginosa pneumonia. (A and B) Numbers of bacteria in (A) BAL and (B) lungs of WT and NIrc4 ${ }^{-/-}$mice. (C) IL-1 $\beta$ concentration and (D) numbers of bacteria in BAL of mice treated with DMSO and caspase-1 inhibitor (CASPinh) after PAK infection. (E) Immune cell populations after infection. PMN, polymorphonuclear neutrophils. (F) H\&E (original magnification, $\times 100)$ and $(\mathbf{G})$ TUNEL (original magnification, $\times 400)$ staining of PAK-infected lungs. (H) Mortality 18 hours after infection $\left(10^{8} \mathrm{CFU} / \mathrm{mouse} ; n=15\right)$. Data are representative of at least 2 independent experiments.

Inbibition of the NLRC4 inflammasome improves clearance of $P$. aeruginosa. Flagellin induces caspase- 1 cleavage of pro-IL- $1 \beta$ by activating the NLRC4 inflammasome $(16,18,19)$. Therefore, we hypothesized that inhibition of the NLRC4 inflammasome would improve clearance of $P$. aeruginosa during acute pneumonia (19). Significantly fewer bacteria were recovered from the airways of $\mathrm{Nlrc4}^{-/-}$versus WT mice after an 18 -hour infection with PAK $(P=0.0006)$; however, similar numbers were observed in lung tissue (Figure 3, A and B).

Generation of IL- $1 \beta$ and IL-18 from pro-IL-1 $\beta$ and pro-IL-18 is induced by flagellin recognition by the NLRC 4 inflammasome and resultant activation of caspase- 1 . To confirm the involvement of caspase- 1 in production of IL- $1 \beta$ during PAK pneumonia, mice were treated systemically with a caspase- 1 inhibitor. Treated mice had significantly reduced IL- $1 \beta$ secretion into the airway and PAK numbers recovered from BAL $(P=0.0126$ and $P=0.0221$, respectively; Figure $3, \mathrm{C}$ and D). Caspase- 1 inhibition did not alter the nature of immune cell recruitment into the airways (Figure 3E). Mice treated with the caspase-1 inhibitor also had better preservation of airspaces, reduced tissue damage (as indicated by TUNEL staining), and a trend toward diminished mortality (Figure 3, F-H). Treatment with the caspase-1 inhibitor alone did not result in tissue damage (Supplemental Figure 4A). Improved bacterial clearance and lung pathology was not due to reduced levels of other inflammatory cytokines, such as $\mathrm{KC}$ and
TNF (Supplemental Figure 4B). These data indicate that signaling through the NLRC4 inflammasome interferes with optimal bacterial clearance from the airway.

Recent reports have highlighted caspase-11 as an upstream, Trif-dependent regulator of caspase- 1 activation in response to certain types of bacteria $(42,43)$. In vitro studies suggest no role for caspase- 11 in $P$. aeruginosa-mediated induction of IL- $1 \beta$ signaling. To confirm these data in vivo, we infected C57BL/6 mice and 129S6 mice, which lack caspase-11. Similar numbers of bacteria were recovered from BAL and lung of the 2 mouse strains (Supplemental Figure 4, C and D). Levels of IL- $1 \beta$ in the airway were also similar between groups (Supplemental Figure 4E). Therefore, we conclude that in vivo caspase-11 does not play a role in PAK induction of the NLRC4 inflammasome.

Regulation of inflammasome-dependent signaling by type I IFN. Our results suggest that inflammasome activation in AM, as measured by IL-1 $\beta$ and IL-18, is likely to be responsible for much of the pathology associated with acute PAK infection. Previous studies using mice lacking IL-1R or treated with IL-1R antagonists indicated that IL-1 signaling contributes to the pathology induced by PA103, a nonmotile strain with upregulated type III toxin production (29). To better establish the relationship between IL-1 $\beta$ production and lung damage, we studied the effect of type I IFN signaling, which regulates pro-IL-1 $\beta$ production, on PAK clearance $(44,45)$. We confirmed the expected association between type I 

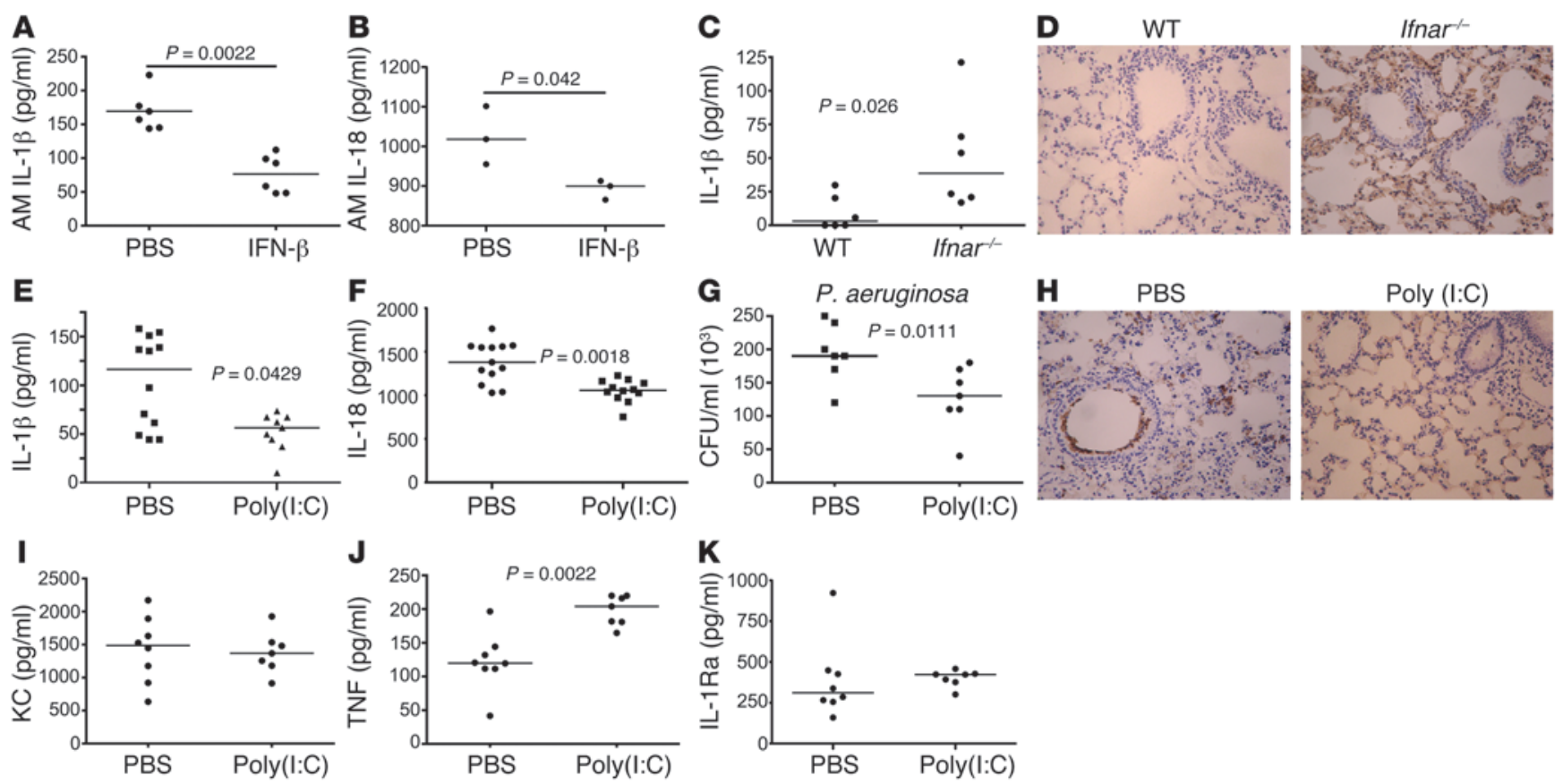

\section{Figure 4}

Type I IFN regulation of inflammasome-dependent cytokines. (A and B) Secretion of (A) IL-1 $\beta$ and (B) IL-18 from AMs pretreated with PBS or type I IFN (IFN- $\beta ; 5 \mu \mathrm{g} / \mathrm{ml}$ ) in vitro. (C) IL-1 $\beta$ in BAL of uninfected WT and Ifnar ${ }^{-1}$ mice. (D) TUNEL staining of uninfected WT and Ifnar ${ }^{-1-}$ mice (original magnification, $\times 400$ ). ( $E$ and $\mathbf{F}$ ) Levels of $(\mathbf{E}) \mathrm{IL}-1 \beta$ and $(\mathbf{F}) \mathrm{IL}-18$ in BAL of PAK-infected WT mice pretreated with PBS or poly(I:C). (G) Numbers of bacteria in BAL of PAK-infected WT mice pretreated with PBS or poly(I:C). (H) TUNEL staining of infected mice pretreated with PBS or poly $(\mathrm{I}: \mathrm{C})$ (original magnification, $\times 400$ ). (I-K) Levels of (I) KC, (J) TNF, and (K) IL-1Ra in BAL of infected mice. Data are representative of at least 2 independent experiments.

IFNs and IL-1 $\beta$ in the context of PAK infection in vitro by demonstrating that PAK induced significantly less IL- $1 \beta$ secretion from primary murine AMs that were pretreated overnight with type I IFN (IFN- $\beta ; 5 \mu \mathrm{g} / \mathrm{ml})$ compared with PBS $(P=0.0022$; Figure 4A). Type I IFN pretreatment also showed a similar effect on IL-18 production $(P=0.042$; Figure 4B).

The relationship of type I IFN, IL- $1 \beta$, and lung pathology was also observed in vivo. Even without infection, IL-1 $\beta$ levels were significantly elevated in the BAL of IFN- $\alpha / \beta$ receptor-null (Ifnar $\left.{ }^{--}\right)$ mice $(P=0.026)$ and correlated with positive TUNEL staining in the lung (Figure 4, C and D). Stimulation of type I IFN signaling with poly(I:C) in WT mice 24 hours prior to bacterial infection inhibited IL-1 $\beta$ and IL-18 secretion into the BAL $(P=0.0429$ and $P=0.0018$, respectively), improved PAK clearance from the airway, and reduced tissue damage, as assessed by TUNEL staining (Figure 4, E-H, and refs. 46-48). The improved outcome could not be attributed to a reduction in either $\mathrm{KC}$ or TNF or to an increase in IL-1Ra (Figure 4, I-K). Immune cell numbers in BAL after poly(I:C) pretreatment and PAK infection have previously been shown to be similar to those of PBS-pretreated animals $(1,2,46)$, which suggests that the beneficial effects correlated primarily with decreased inflammasome activity, as measured by IL- $1 \beta$ and IL-18.

Inbibition of IL-1 or IL-18 signaling improves P. aeruginosa clearance. The key components of inflammasome signaling, IL-1 $\beta$ and IL-18, both participate in discrete proinflammatory signaling cascades. To address whether these pathways are independently responsible for the delayed bacterial clearance associated with activation of the inflammasome or caspase-1, we infected mice lacking the recep-

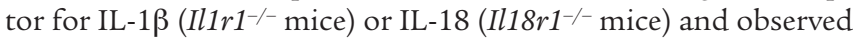

improved clearance of PAK from the airways in both groups compared with WT controls $(P=0.0317$ and $P=0.0087$, respectively; Figure 5, A and B). These data indicate that each of these cytokines contributes to impaired bacterial clearance during PAK pneumonia. Whereas immune cell recruitment was not altered in Ill $\mathrm{r}^{-/-}$mice compared with WT controls, more AMs were found in the airway of $I l 18 \mathrm{r1}^{-/-}$versus WT mice $(P=0.026$; Figure 5, C and D). Interestingly, despite an increase in AM numbers, inhibition of IL-18 signaling resulted in improved clearance. Thus, the lung damage associated with acute PAK infection correlated with caspase- 1 activity and with IL- $1 \beta$ and IL-18 production, but not with the numbers of recruited neutrophils or expression of other inflammatory cytokines.

\section{Discussion}

The host response to inhaled extracellular pathogens, such as $P$. aeruginosa and other opportunists, involves the cooperative responses of epithelial cells, pulmonary DCs, T cells, recruited neutrophils, and AMs. In the present study, we sought to determine whether specific host signaling cascades are responsible for the pathology associated with acute $P$. aeruginosa infection. While activation of NF-kB-mediated proinflammatory chemokines and cytokines is a widely shared response to bacterial PAMPs, stimulation of inflammasome signaling is more stringently regulated. Inflammasome signaling induced by $P$. aeruginosa flagella resulted in IL-1 $\beta$ and IL-18 production primarily from the AMs that was not only dispensable for bacterial clearance, but also associated with excessive lung pathology and increased mortality. Strategies to diminish production of these cytokines in mice by using fla- 

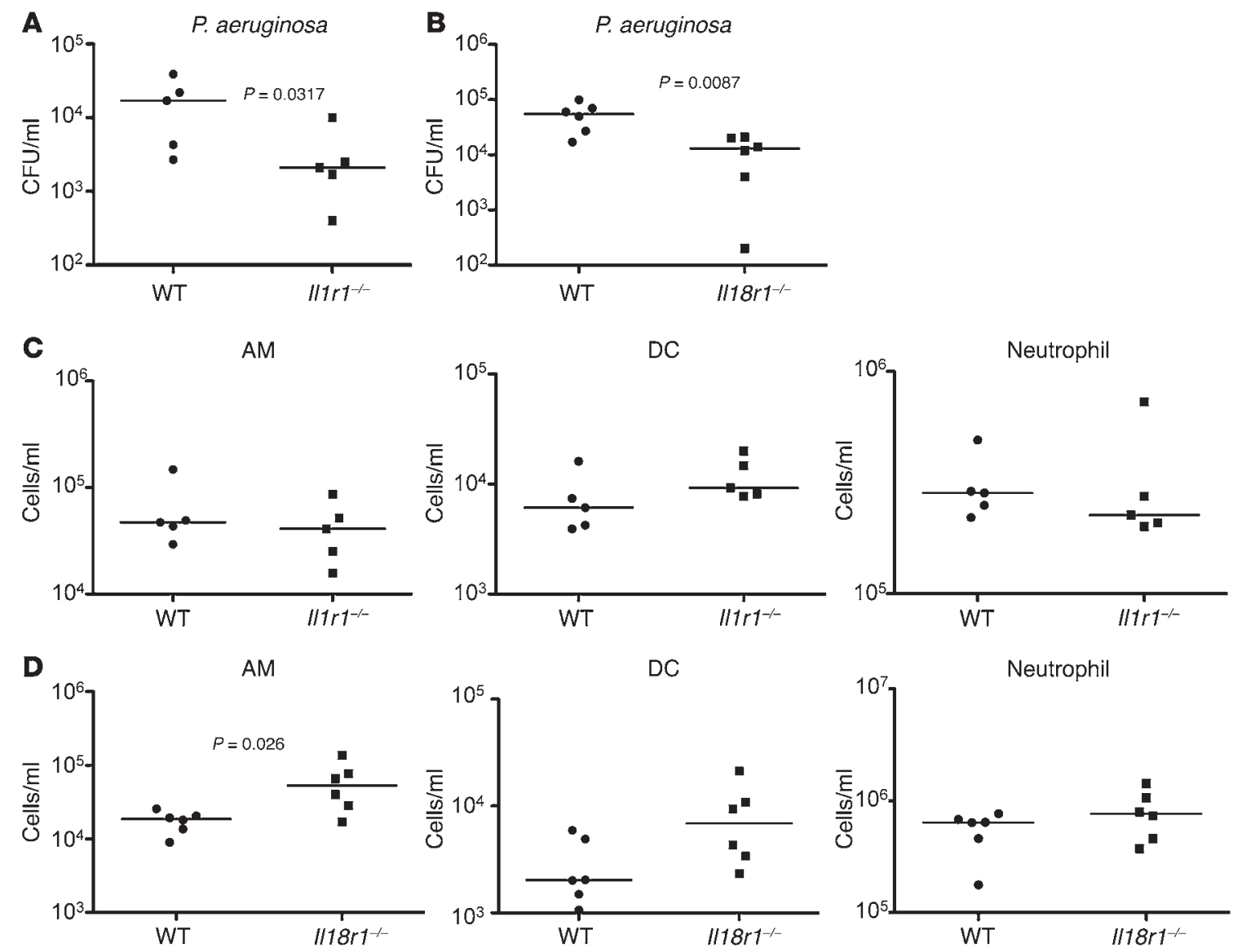

Figure 5

IL-1 and IL-18 inhibition improves $P$. aeruginosa clearance. (A and B) Numbers of bacteria in BAL of (A) $/ / 1 r 1^{-/-}$and (B) $/ / 18 r 1^{-/-}$mice compared with WT. (C and D) Immune cell populations in BAL of (C) $/ / 1 \mathrm{r}^{-1-}$ and (D) $/ / 18 r 1^{-1-}$ mice after infection, compared with WT. Data are representative of at least 2 independent experiments.

gellin-deficient bacteria, depleting AMs, increasing type I IFN signaling, inhibiting caspase-1, knocking out NLRC4, or inhibiting IL-1 or IL-18 signaling resulted in improved bacterial clearance, decreased mortality, and less pulmonary damage. Morbidity and mortality was not associated with the extent of neutrophil recruitment or the mobilization of DCs or T cells.

For extracellular pathogens such as $P$. aeruginosa, bacterial clearance is dependent upon neutrophil phagocytosis and killing, as the organisms do not accumulate within macrophages. Clearance of intracellular organisms such as Salmonella or Burkbolderia requires induction of caspase-1-dependent pyroptosis to release sequestered bacteria from AMs and allow for neutrophil-mediated killing $(3-7,15,49)$. It is unclear whether host defense against acute $P$. aeruginosa infection requires inflammasome activation. Instead, we observed decreased bacterial clearance and increased cytotoxicity in the lung specifically associated with the inflammasome-dependent products IL-1 $\beta$ and IL- 18 .

AM depletion was accompanied by compensatory changes in other cytokines and chemokines, including GM-CSF and components of the IL-17 pathway. An increase in GM-CSF production, most likely a product of airway epithelial cells, stimulated the phagocytic capabilities of the recruited neutrophils and likely added to the clearance of organisms $(8-13,50)$. In contrast to pre- vious reports suggesting that beneficial effects of IL-1 blockade in bacterial pneumonia are primarily due to diminished recruitment of neutrophils, our data suggest that it is the nature of the proinflammatory signaling that is important for limiting pulmonary damage, not simply the number of neutrophils found in the lung $(14-22,26,29)$. AM depletion also resulted in decreased IL-17, a cytokine that enhanced the clearance of extracellular pathogens, as confirmed in $\mathrm{Cd} 4^{-/-}$mice and in mice treated with anti-IL-17 $(1,2,23-29,39)$. However, despite the association of decreased IL-17 with AM depletion, bacterial clearance was significantly increased. This suggests that inflammasome activation, and secretion of IL-1 $\beta$ and IL-18, was biologically more significant than the loss of IL-17 signaling in acute $P$. aeruginosa pneumonia.

We were also able to demonstrate the participation of the type I IFN cascade in the regulation of IL- $1 \beta$ and IL-18. Type I IFNs are produced primarily by DCs in the respiratory tract and have become increasingly appreciated as an important component of innate immune defenses to extracellular pathogens, including $P$. aeruginosa (3-7, 30-33, 46, 51-54). Strategies to stimulate the type I IFN cascade decreased IL-1 $\beta$ and IL-18 production, enhanced $P$. aeruginosa clearance, and diminished lung damage. Thus, type I IFN regulation of the inflammasome appears to function to ameliorate pulmonary damage from infection. 
$P$. aeruginosa flagella are recognized as important in pathogenesis, but were not previously associated with detrimental host responses $(3,8-13,32,34,55)$. Our data suggest that the established role of flagella in nonopsonic phagocytosis delivers flagellated organisms to AMs, where, upon ingestion, the flagella activate the NLRC4 inflammasome (14-22, 34, 35). Murine epithelial cells were found to produce IL- $1 \beta$ in response to both WT and fliC PAK; hence, other effectors, such as type III secretion system components or pili, may be involved in epithelial signaling $(16,19$, $36,56-58)$. This hypothesis is supported by a recent study in which $P$. aeruginosa lacking the type III secretion system stimulated less caspase- 1 activation, IL- $1 \beta$ secretion, and neutrophil recruitment after only 4 hours of infection $(37-41,59)$. The contribution of the NLRC4 inflammasome in stromal cells as well as AMs was previously shown to be important for $P$. aeruginosa clearance, although this was in mice with a different genetic background $(19,60)$. Clearance of another Gram-negative bacteria, Klebsiella pneumoniae, was also positively influenced by the presence of the NLRC4 inflammasome, although significant differences in bacterial clearance were only observed after a 48-hour infection $(14,42,43)$. Thus, additional effectors of inflammasome signaling are likely to contribute to bacterial clearance through other mechanisms.

The pathogenesis of acute $P$. aeruginosa pneumonia involves multiple interactions of both the organism and host immune effectors. Our data suggest that the ability of $P$. aeruginosa to activate the NLRC4 inflammasome-mediated production of IL- $1 \beta$ and IL-18 is responsible for a substantial amount of the pathology associated with acute pneumonia. Caspase-1, IL-1R, and IL-18R are potential targets to limit pathologic consequences of infection and improve bacterial clearance. As it is difficult, if not impossible, to predict which patients will become at risk for these types of opportunistic infections, preventative therapy targeted at the organism is problematic. However, in specific settings, it should be possible to modulate components of inflammasome activation in the airway, with either caspase-1 inhibition or stimulation of regulatory pathways, as shown herein. Inhibition of specific pathological inflammatory responses in the setting of acute pneumonia, as is the norm in other causes of inflammation, should provide a useful adjuvant to traditional antimicrobial therapy.

\section{Methods}

Bacterial strains. The P. aeruginosa strains PAK (WT), fliC PAK, and motAB PAK (provided by S. Lory, Harvard University, Boston, Massachusetts, USA), as well as PAK gfp (provided by M. Ulanova, Lakehead University, Thunder Bay, Ontario, Canada), were grown on LB. Plate cultures were grown at $37^{\circ} \mathrm{C}$. Growth rates of WT PAK, fliC PAK, and motAB PAK did not differ (Supplemental Figure 5).

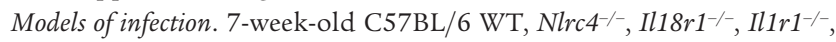
$\mathrm{Cd}^{-/-}$, or Ifnar $/-$mice were intranasally inoculated with $50 \mu \mathrm{l} P$. aeruginosa $\left(1 \times 10^{7} \mathrm{CFU}\right.$, or $1 \times 10^{8} \mathrm{CFU}$ for mortality), as previously described $(29,46)$.

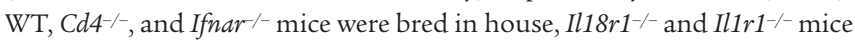
were bought from Jackson Laboratories, and $\mathrm{Nlrc}^{-/-}$mice were provided by Genentech. Control mice received $50 \mu$ l PBS. Mice were pretreated i.p. with the caspase- 1 inhibitor Ac-YVAD-AOM (30 $\mu \mathrm{g} /$ mouse; EMD Biosciences) or neutralizing antibodies to GM-CSF (50 $\mu \mathrm{g}$ /mouse; eBioscience), IL-6 (100 $\mu \mathrm{g} /$ mouse; eBioscience), or IL-17 (10 $\mu \mathrm{g} /$ mouse; R\&D Biosystems)
1 hour prior to infection. Control mice were treated with DMSO and rat IgG for caspase inhibitor and cytokine antibody experiments, respectively. Clodronate depletion of AM was done as described previously $(44,45,61)$. BAL fluid was harvested 18 hours after infection and used to quantify immune cell populations, cytokine expression, and bacteria CFU. Histology and TUNEL staining was performed by Columbia University Molecular Pathology core on tissues fixed in 4\% paraformaldehyde. 6 independent, blinded reviewers assigned each image a lung injury score from 0 (healthy) to 5 (injured). Primary nasal epithelial cells were isolated and cultured as previously described $(46-48,62)$. AMs, the primary cell type recovered from uninfected BAL (>85\%), were resuspended in RPMI 1640 plus 10\% fetal bovine serum. Epithelial cells and AM were pretreated with LPS $(50 \mathrm{ng} / \mathrm{ml})$ overnight prior to a 2-hour infection (MOI 10).

FACS analysis. Enumeration of neutrophil (MHCII-Ly6 $\left.6^{+}\right), \mathrm{AM}$ $\left(\mathrm{CD} 11 \mathrm{c}^{+} \mathrm{MHCII}^{\mathrm{lo}}\right)$, and $\mathrm{DC}\left(\mathrm{CD} 11 \mathrm{c}^{+} \mathrm{MHCII}^{+}\right)$populations was performed as previously described (46). Antibody to CD4 (clone RM4.5; eBioscience) and IL-17A (clone eBio17B7; eBioscience) were used to identify CD4 $\left(\mathrm{CD} 4{ }^{+} \mathrm{CD} 11 \mathrm{c}^{-}\right)$and Th17 $\left(\mathrm{IL}-17^{+} \mathrm{CD} 4{ }^{+} \mathrm{CD} 11 \mathrm{c}^{-}\right)$cell populations. Intracellular staining for IL-17A was done after simulation with phorbol 12-myristate 13-acetate and ionomycin (Calbiochem) in the presence of Brefeldin A (eBioscience), as previously described (41). Cellular permeabilization and intracellular staining was done using a commercially available kit (eBioscience) according to the manufacturer's instructions.

ELISA and immunoblotting. BAL or cell free supernatant from AMs or epithelial culture was analyzed for cytokine and chemokine content by ELISA (R\&D Biosystems or eBioscience) according to the manufacturer's instructions. Caspase-1 activation was monitored with antibody to caspase-1 p10 (Santa Cruz Biotechnology Inc.). Phosphorylation of STAT3 in cells recovered from BAL was detected using phospho-STAT3 (Y705; Cell Signaling) and $\beta$-actin (Sigma-Aldrich) antibodies followed by secondary antibodies conjugated to horseradish peroxidase (Santa Cruz Biotechnology Inc.). Protein separation, transfer, and immunoblotting were performed as described previously (52).

Statistics. Significance of data was determined using a nonparametric Mann-Whitney test. For experiments with more than 1 comparison, we used a nonparametric Kruskal-Wallis test followed by post-hoc Dunn test to correct for multiple comparisons. Mortality data were compared using Fisher exact test. Statistics were performed with GraphPad Prism software; a $P$ value less than 0.05 was considered significant. In vivo data are presented as individual points with bars representing median values; in vitro data are presented as bar graphs (mean $\pm \mathrm{SD}$ ).

Study approval. Animal experiments were performed in accordance with the guidelines of the IACUC at Columbia University (protocol no. AAAC3059).

\section{Acknowledgments}

This work was supported by NIH grant R01HL073989 to A.S. Prince and by a Parker B. Francis Fellowship to T.S. Cohen.

Received for publication August 1, 2012, and accepted in revised form January 17, 2013.

Address correspondence to: Alice S. Prince, Columbia University, Department of Pediatrics, 650 West 168th Street, Black Building 418, New York, New York 10032, USA. Phone: 212.305.4193; Fax: 212.305.2284; E-mail: asp7@columbia.edu.
1. Zhuo $\mathrm{H}$, et al. Increased mortality of ventilated patients with endotracheal Pseudomonas aeruginosa without clinical signs of infection. Crit Care Med. 2008;36(9):2495-2503.
2. Richards MJ, Edwards JR, Culver DH, Gaynes RP. Nosocomial infections in medical intensive care units in the United States. National Nosocomial Infections Surveillance System. Crit Care Med. 1999;
27(5):887-892.

3. Adamo R, Sokol S, Soong G, Gómez MI, Prince A. Pseudomonas aeruginosa flagella activate airway epithelial cells through asialoGM1 and toll-like recep- 
tor 2 as well as toll-like receptor 5. Am J Respir Cell Mol Biol. 2004;30(5):627-634.

4. Tseng J, Do J, Widdicombe JH, Machen TE. Innate immune responses of human tracheal epithelium to Pseudomonas aeruginosa flagellin, TNF-alpha, and IL-1beta. Am J Physiol Cell Physiol. 2006;290(3):C678-C690.

5. Poynter ME, Irvin CG, Janssen-Heininger YMW. A prominent role for airway epithelial NF-kappa $B$ activation in lipopolysaccharide-induced airway inflammation. J Immunol. 2003;170(12):6257-6265.

6. Smith SM, Lee DK, Lacy J, Coleman DL. Rat tracheal epithelial cells produce granulocyte/macrophage colony-stimulating factor. Am J Respir Cell Mol Biol. 1990;2(1):59-68

7. Cobb LM, MychaleckyjJC, Wozniak DJ, López-Boado YS. Pseudomonas aeruginosa flagellin and alginate elicit very distinct gene expression patterns in airway epithelial cells: implications for cystic fibrosis disease. J Immunol. 2004;173(9):5659-5670.

8. Takeuchi $\mathrm{O}$, et al. Discrimination of bacterial lipoproteins by Toll-like receptor 6. Int Immunol. 2001; 13(7):933-940.

9. Takeuchi O, et al. Cutting edge: role of Toll-like receptor 1 in mediating immune response to microbial lipoproteins. J Immunol. 2002;169(1):10-14.

10. Aliprantis AO, et al. Cell activation and apoptosis by bacterial lipoproteins through toll-like receptor-2. Science. 1999;285(5428):736-739.

11. Schwandner R, Dziarski R, Wesche H, Rothe M, Kirschning CJ. Peptidoglycan- and lipoteichoic acid-induced cell activation is mediated by toll-like receptor 2.J Biol Chem. 1999;274(25):17406-17409.

12. Poltorak A, et al. Defective LPS signaling in $\mathrm{C} 3 \mathrm{H} /$ $\mathrm{HeJ}$ and $\mathrm{C} 57 \mathrm{BL} / 10 \mathrm{ScCr}$ mice: mutations in Tlr4 gene. Science. 1998;282(5396):2085-2088.

13. Hayashi F, et al. The innate immune response to bacterial flagellin is mediated by Toll-like receptor 5 . Nature. 2001;410(6832):1099-1103.

14. Cai S, Batra S, Wakamatsu N, Pacher P, Jeyaseelan S. NLRC4 inflammasome-mediated production of IL-1 $\beta$ modulates mucosal immunity in the lung against gram-negative bacterial infection. J Immunol. 2012;188(11):5623-5635.

15. Miao EA, et al. Caspase-1-induced pyroptosis is an innate immune effector mechanism against intracellular bacteria. Nat Immunol. 2010; 11(12):1136-1142.

16. Sutterwala FS, et al. Immune recognition of Pseudomonas aeruginosa mediated by the IPAF/NLRC4 inflammasome. J Exp Med. 2007;204(13):3235-3245.

17. Ren T, Zamboni DS, Roy CR, Dietrich WF, Vance RE. Flagellin-deficient Legionella mutants evade caspase-1- and Naip5-mediated macrophage immunity. PLoS Pathog. 2006;2(3):e18.

18. Mariathasan S, et al. Differential activation of the inflammasome by caspase- 1 adaptors ASC and Ipaf. Nature. 2004;430(6996):213-218.

19. Miao EA, Ernst RK, Dors M, Mao DP, Aderem A. Pseudomonas aeruginosa activates caspase 1 through Ipaf. Proc Natl Acad Sci U S A. 2008;105(7):2562-2567.

20. Bergsbaken T, Cookson BT. Macrophage activation redirects yersinia-infected host cell death from apoptosis to caspase-1-dependent pyroptosis. PLoS Pathog. 2007;3(11):e161.

21. Fink SL, Bergsbaken T, Cookson BT. Anthrax lethal toxin and Salmonella elicit the common cell death pathway of caspase-1-dependent pyroptosis via distinct mechanisms. Proc Natl Acad Sci U S A. 2008; 105(11):4312-4317.

22. Fink SL, Cookson BT. Caspase-1-dependent pore formation during pyroptosis leads to osmotic lysis of infected host macrophages. Cell Microbiol. 2006;8(11):1812-1825.

23. Kang M-J, et al. IL-18 induces emphysema, and airway and vascular remodeling via IFN- $\gamma$, IL-17A and IL-13. Am J Respir Crit Care Med. 2012; 185(11):1205-1217

24. Ghose P, et al. The interaction between IL-18 and IL-18 receptor limits the magnitude of protective immunity and enhances pathogenic responses following infection with intracellular bacteria. J Immunol. 2011;187(3):1333-1346

25. Faggioni R, et al. IL-18-binding protein protects against lipopolysaccharide- induced lethality and prevents the development of Fas/Fas ligandmediated models of liver disease in mice. J Immunol. 2001;167(10):5913-5920.

26. Véliz Rodriguez T, et al. Role of toll interleukin-1 receptor (IL-1R) 8, a negative regulator of il-1r/tolllike receptor signaling, in resistance to acute Psendomonas aeruginosa lung infection. Infect Immun. 2012; 80(1):100-109.

27. Rider P, et al. IL-1 and IL-1 recruit different myeloid cells and promote different stages of sterile inflammation. J Immunol. 2011; 187(9):4835-4843.

28. Kolb M, Margetts PJ, Anthony DC, Pitossi F, Gauldie J. Transient expression of IL- $1 \beta$ induces acute lung injury and chronic repair leading to pulmonary fibrosis. J Clin Invest. 2001;107(12):1529-1536.

29. Schultz MJ, et al. Role of interleukin-1 in the pulmonary immune response during Psendomonas aeruginosa pneumonia. Am J Physiol Lung Cell Mol Physiol. 2002;282(2):L285-L290.

30. Singbartl K, et al. Differential effects of kidneylung cross-talk during acute kidney injury and bacterial pneumonia. Kidney Int. 2011;80(6):633-644.

31. Manicone AM, et al. Epilysin (MMP-28) restrains early macrophage recruitment in Pseudomonas aeruginosa pneumonia. JImmunol. 2009;182(6):3866-3876.

32. Morris AE, Liggitt HD, Hawn TR, Skerrett SJ. Role of Toll-like receptor 5 in the innate immune response to acute $P$. aeruginosa pneumonia. Am J Physiol Lung Cell Mol Physiol. 2009;297(6):L1112-L1119.

33. Sun L, et al. Effect of IL-10 on neutrophil recruitment and survival after Pseudomonas aeruginosa challenge. Am J Respir Cell Mol Biol. 2009;41(1):76-84.

34. Mahenthiralingam E, Speert DP. Nonopsonic phagocytosis of Pseudomonas aeruginosa by macrophages and polymorphonuclear leukocytes requires the presence of the bacterial flagellum. Infect Immun. 1995;63(11):4519-4523.

35. Dasgupta $\mathrm{N}$, et al. A four-tiered transcriptional regulatory circuit controls flagellar biogenesis in Pseudomonas aeruginosa. Mol Microbiol. 2003; 50(3):809-824.

36. Labbé K, Saleh M. Cell death in the host response to infection. Cell Death Differ. 2008;15(9):1339-1349.

37. Aujla SJ, et al. IL-22 mediates mucosal host defense against Gram-negative bacterial pneumonia. Nat Med. 2008;14(3):275-281.

38. Dubin PJ, et al. IL-23 Mediated inflammation in Pseudomonas aeruginosa pulmonary infection. Infect Immun. 2012;80(1):398-409.

39. Ye P, et al. Requirement of interleukin 17 receptor signaling for lung CXC chemokine and granulocyte colony-stimulating factor expression, neutrophil recruitment, and host defense.J Exp Med. 2001; 194(4):519-527.

40. Balamayooran T, et al. Receptor-interacting protein 2 controls pulmonary host defense to Escherichia coli infection via the regulation of interleukin-17A. Infect Immun. 2011;79(11):4588-4599.

41. Kimura A, Naka T, Kishimoto T. IL-6-dependent and -independent pathways in the development of interleukin 17-producing T helper cells. Proc Nat Acad Sci U S A. 2007;104(29):12099-12104

42. Rathinam VAK, et al. TRIF licenses caspase-11-dependent NLRP3 inflammasome activation by gram-negative bacteria. Cell. 2012;150(3):606-619.

43. Broz P, et al. Caspase- 11 increases susceptibility to Salmonella infection in the absence of caspase- 1 . Nature. 2012;490(7419):288-291.

44. Guarda G, et al. Type I interferon inhibits interleukin-1 production and inflammasome activation. Immunity. 2011;34(2):213-223.

45. Novikov A, et al. Mycobacterium tuberculosis triggers host type I IFN signaling to regulate IL-1 $\beta$ production in human macrophages. J Immunol. 2011;187(5):2540-2547.

46. Parker D, et al. Induction of type I interferon signaling by Psendomonas aeruginosa is diminished in cystic fibrosis epithelial cells. Am J Respir Cell Mol Biol. 2012;46(1):6-13.

47. Oganesyan $\mathrm{G}$, et al. Critical role of TRAF3 in the toll-like receptor-dependent and -independent antiviral response. Nature. 2006;439(7073):208-211.

48. Monroe KM, McWhirter SM, Vance RE. Identification of host cytosolic sensors and bacterial factors regulating the type I interferon response to Legionella pneumophila. PLoS Pathog. 2009;5(11):e1000665.

49. Ceballos-Olvera I, Sahoo M, Miller MA, Barrio LD, Re F. Inflammasome-dependent pyroptosis and IL-18 protect against Burkholderia pseudomallei lung infection while IL-1 $\beta$ is deleterious. PLoS Pathog. 2011;7(12):e1002452.

50. Saba S, Soong G, Greenberg S, Prince A. Bacterial stimulation of epithelial G-CSF and GM-CSF expression promotes PMN survival in CF airways. Am J Respir Cell Mol Biol. 2002;27(5):561-567.

51. Parker D, et al. Streptococcus pneumoniae DNA initiates type $\mathrm{I}$ interferon signaling in the respiratory tract. MBio. 2011;2(3):e00016-11.

52. Martin FJ, et al. Staphylococcus aureus activates type I IFN signaling in mice and humans through the $\mathrm{Xr}$ repeated sequences of protein A. J Clin Invest. 2009;119(7):1931-1939.

53. Carrigan $\mathrm{SO}$, et al. IFN regulatory factor 3 contributes to the host response during Pseudomonas aeruginosa lung infection in mice. J Immunol. 2010; 185(6):3602-3609.

54. Power MR, Li B, Yamamoto M, Akira S, Lin T-J. A role of Toll-IL-1 receptor domain-containing adaptor-inducing IFN-beta in the host response to Pseudomonas aeruginosa lung infection in mice. J Immunol. 2007;178(5):3170-3176.

55. Balloy V, et al. The role of flagellin versus motility in acute lung disease caused by Psendomonas aeruginosa. J Infect Dis. 2007;196(2):289-296.

56. Arlehamn CSL, Evans TJ. Pseudomonas aeruginosa pilin activates the inflammasome. Cell Microbiol. 2011;13(3):388-401.

57. Zhao Y, et al. The NLRC4 inflammasome receptors for bacterial flagellin and type III secretion apparatus. Nature. 2011;477(7366):596-600

58. Miao EA, Andersen-Nissen E, Warren SE, Aderem A. TLR5 and Ipaf: dual sensors of bacterial flagellin in the innate immune system. Semin Immunopathol. 2007;29(3):275-288.

59. Wangdi T, Mijares LA, Kazmierczak BI. In vivo discrimination of type 3 secretion system-positive and -negative Pseudomonas aeruginosa via a caspase-1-dependent pathway. Infect Immun. 2010; 78(11):4744-4753.

60. Franchi L, et al. Critical role for Ipaf in Psendomonas aeruginosa-induced caspase- 1 activation. Eur J Immunol. 2007;37(11):3030-3039.

61. Martin FJ, Parker D, Harfenist BS, Soong G, Prince A. Participation of CD11c(+) leukocytes in methicillin-resistant Staphylococcus aureus clearance from the lung. Infect Immun. 2011;79(5):1898-1904.

62. Antunes MB, et al. Murine nasal septa for respiratory epithelial air-liquid interface cultures. BioTechniques. 2007;43(2):195-204. 